\title{
Effective Irradiation of a Metastatic Region of the Lung in a Patient with Extraskeletal Myxoid Chondrosarcoma
}

\author{
Yutaka Mizushima, Masashi Fusita, Hitoshi Hirata, Kuninori Nomura*, Hisao Matsu**, \\ Kazuhiro MATSUI $* * *$ and Masashi KoBAYASHI
}

\begin{abstract}
Complete obstruction of the right main bronchus occurred in a 76-year-old woman due to pulmonary metastasis from the extraskeletal myxoid chondrosarcoma (EMC) which had been detected 5 years earlier. Treatment with Nd-YAG laser and biopsy forceps opened up the lumen of the right main bronchus $90 \%$, and then $\mathrm{X}$-irradiation was given toward the remaining tumor. The tumor disappeared almost completely following radiotherapy and such a state had lasted for several years until she died of old age at the age of 81 . We judged that radiotherapy had been effective for this tumor. To date, the information concerning the effect of radiotherapy on this disease is insufficient.
\end{abstract}

(Internal Medicine 37: 625-627, 1998)

Key words: radiosensitivity, radiotherapy, soft tissue sarcoma

\section{Introduction}

Extraskeletal chondrosarcomas were first described in 1953 by Stout and Verner (1), and Enzinger and Shiraki (2) first defined the myxoid variant in 1972 as a distinct clinicopathologic entity. Extraskeletal myxoid chondrosarcoma (EMC) is a rare, low-grade soft tissue sarcoma usually arising in the musculature of the extremities (2-9). This neoplasm is characterized histologically by a mixture of cellular and myxoid stromal components. The tumors grow very slowly, so patients often survive more than ten years after diagnosis. However, metastases predominantly to the lung develop in almost all cases over a period of several years. There are a few reports which have shown chemotherapy to be completely ineffective for this disease $(5,7,8)$, but almost no information is available on the effect of radiotherapy (5). We describe here a case with EMC, in whom radiation therapy was very effective for a metastatic region of the lung.

\section{Case Report}

A 76-year-old woman was admitted to the Department of Internal Medicine on July 3, 1992 because of a feeling of tightness in the chest, dyspnea, cough, and sputum. At the age of 67 , she first noticed a hard mass in her left buttock and subsequently three masses in her left lower extremity. At the age of 71, she underwent resection of the mass in her left buttock in the Department of Orthopedic Surgery, and a diagnosis of EMC was made (Fig. 1). At that time, a mass in the right lung was detected on the chest roentgenograms, but no treatment was employed for it. At the age of 73, three tumor masses in her left lower extremity were resected, but there already existed masses in her right lower extremity at that time. Examinations on admission revealed that her symptoms were due to complete obstruction of the right main bronchus caused by the mass which had been detected 5 years earlier (Figs. 2A, 3A). Partial pressure of oxygen $\left(\mathrm{PaO}_{2}\right)$ in room air was normal of 79.8 torr, but the forced vital capacity was severely impaired (\% predicted forced vital capacity $(\% \mathrm{FVC})=38.3 \%)$. Treatment with Nd-YAG laser and biopsy forceps was employed three times (July 8, July 17, and September 2), and approximately $90 \%$ opening of the right main bronchus was obtained. But the orifice of the right upper lobe remained closed. The pathologic diagnosis of biopsy specimens in the right main bronchus was EMC (data not shown). Consequently, her pulmonary function improved: forced vital capacity (FVC) from $0.76 l$ to $1.60 l$ and forced expiratory volume one second $\left(\mathrm{FEV}_{1}\right)$ from $0.60 l$ to 1.30 $l$. She was temporarily discharged on September 10. Fiberoptic bronchoscopy a month after the last YAG-laser treatment showed the smooth mucosa with residual tumor in the right

From the First Department of Internal Medicine, *the Department of Radiology, **the Department of Orthopedic Surgery and ***the First Department of Pathology, Toyama Medical and Pharmaceutical University, Toyama

Received for publication January 19, 1998; Accepted for publication April 28, 1998

Reprint requests should be addressed to Dr. Yutaka Mizushima, Department of Geriatrics, Hirosaki University School of Medicine, 5 Zaifu-cho, Hirosaki 036-8216 


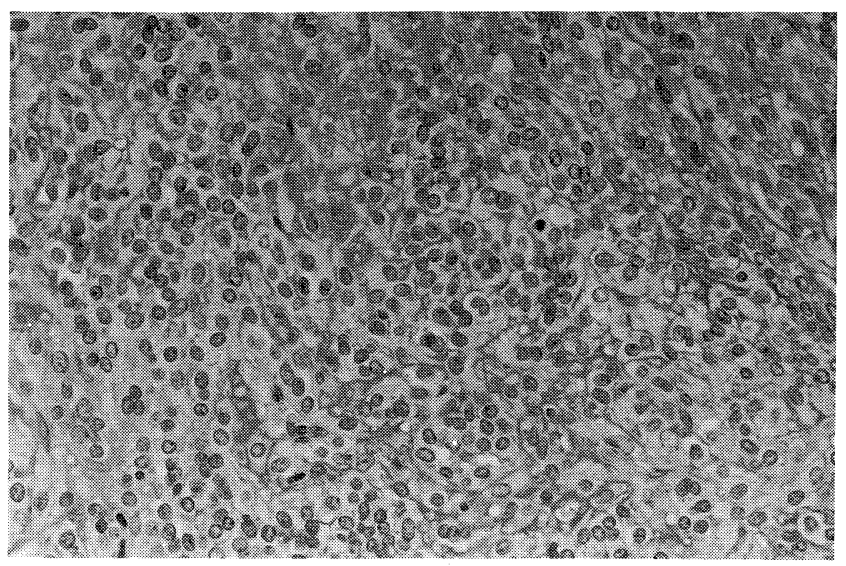

Figure 1. Typical histologic picture of EMC showing a myxoid component and spindle-shaped cells (HE stain, $\times 200$ ).

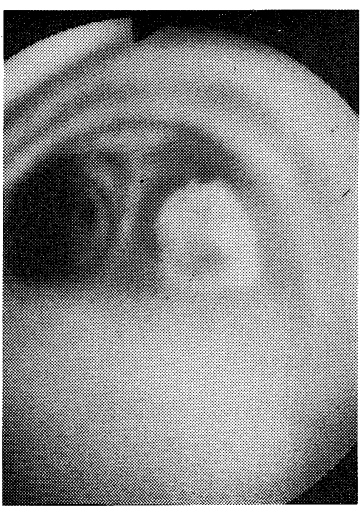

A

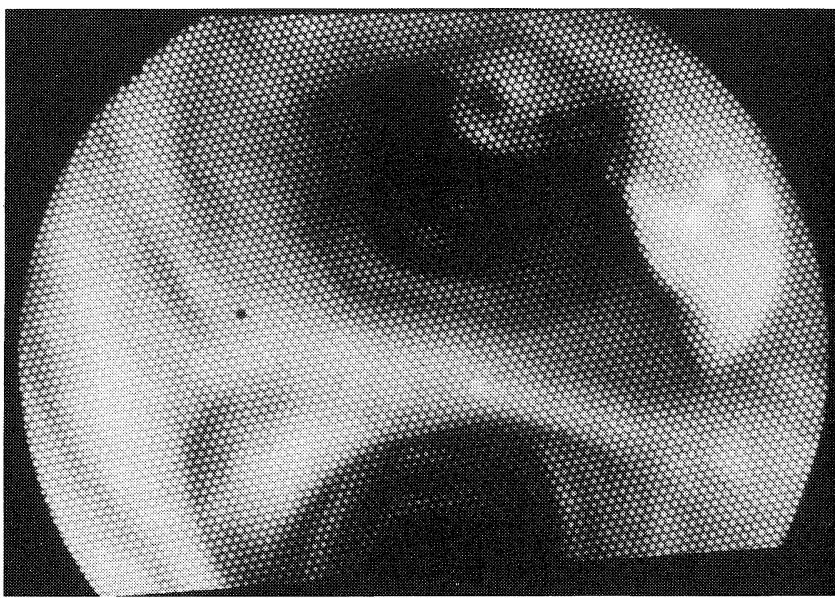

B

Figure 2. A) Fiberoptic bronchoscopy on admission (July 6, 1992) revealed a tumor obstructing the right main bronchus. B) Fiberoptic bronchoscopy (October 10, 1992) showed the smooth mucosa with residual tumor in the right main bronchus. C) Fiberoptic bronchoscopy (April 23, 1997) revealed no tumor in the right main bronchus and complete closure of the orifice of the right upper lobe.
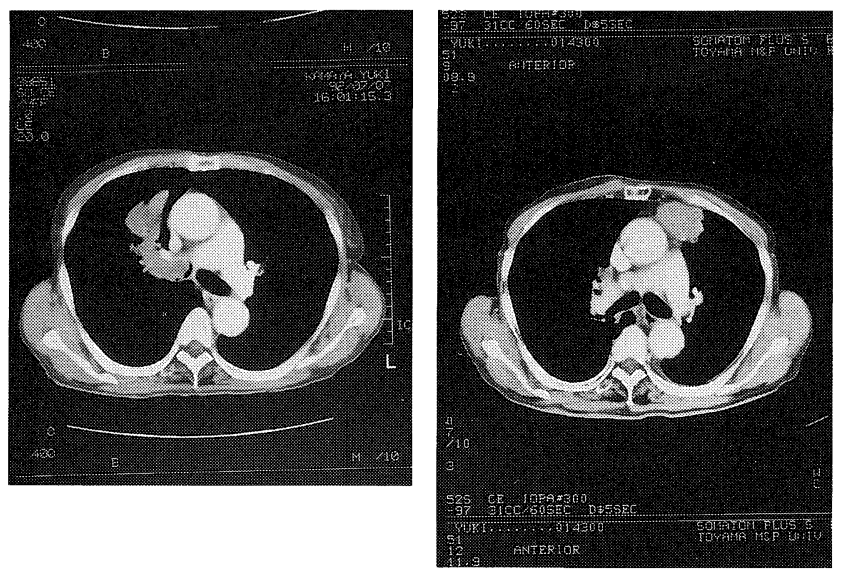

A

C

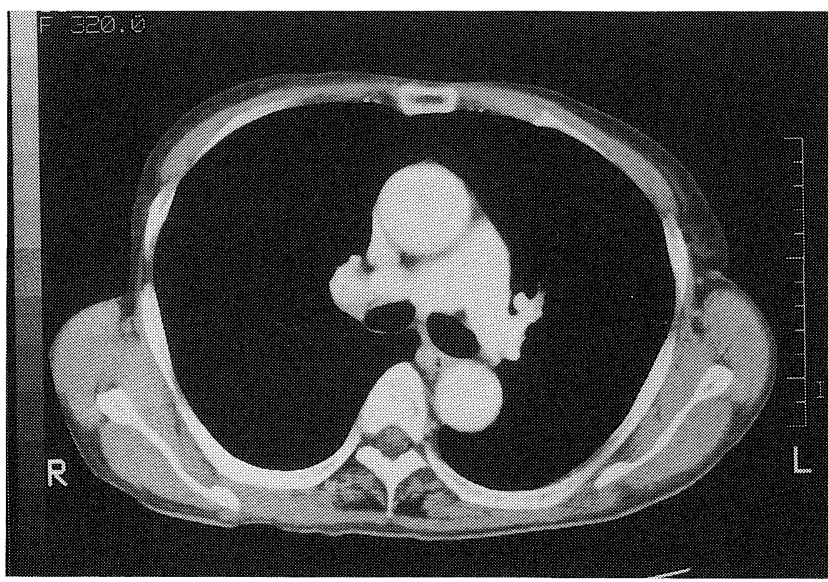

B

Figure 3. A) Chest CT on admission (July 7, 1992) revealed a tumor invading the lumen of the right main bronchus. B) Chest CT (February 4, 1993) indicated the effectiveness of radiation therapy. C) Chest CT (February 20,1997) revealed no tumor in the right main bronchus and a new metastatic region in the left upper lung field.

main bronchus (Fig. 2B).

She was readmitted on November 24 to treat the tumor which remained both inside and outside of the right main bronchus. She was treated with $\mathrm{X}$-irradiation at a dose of $78 \mathrm{~Gy}$ (2 Gy/day) between December 7 and February 9, 1993. The outline of the tumor shadow became clear and its size gradually decreased on the chest roentgenograms, and chest computed tomography (CT) (February 4) indicated that radiation therapy was effective (Fig. 3B). In March, three tumor masses in her right lower extremity were resected and she was discharged on April 4, 1993.

Thereafter she continued to complain of cough and sputum, but not of dyspnea. She was followed in the outpatient clinic for several years, but she gradually became weaker. She was hospitalized on June 5, 1997 and died of old age at 81 years old on July 15. Total clinical course of the disease since she had first 


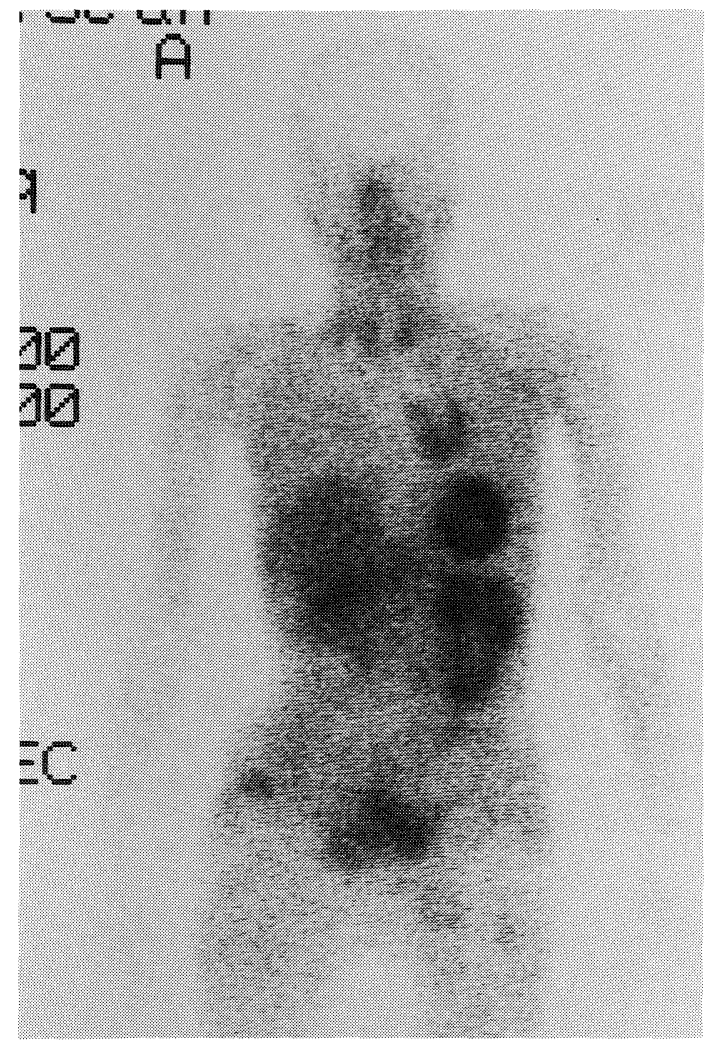

Figure 4. ${ }^{201} \mathrm{Tl}$ whole body scan (May 22, 1997) revealed no abnormal uptake in the right hilar region, but an abnormal accumulation of radioactivity was seen in a new metastatic region of the left lung.

noticed an abnormal body mass was approximately 14 years.

Roentgenographically, the tumor shadow in the right region disappeared almost completely following radiotherapy and such a state had lasted for several years until she died (Fig. 3C). The result of the latest fiberoptic bronchoscopy examination (April 23, 1997) revealed no presence of any tumor in the right main bronchus and complete closure of the orifice of the right upper lobe (Fig. 2). The thallium-201 (T1-201) whole body scan (May 22, 1997) also revealed no abnormal uptake in the right hilar region, although an abnormal accumulation of radioactivity was detected in a new metastatic region of the left lung (Fig. 4). Taken altogether, we judged that radiotherapy had been effective.

\section{Discussion}

We have shown in this report that radiotherapy may be effective for the local control of EMC. This disease is relatively rare, so there are few reports on the therapy for this disease. Traditionally, surgical resection has been the first choice, and the role of radiotherapy or systemic chemotherapy has not been well defined. Several investigators $(5,7,8)$ have reported that chemotherapy is ineffective for this type of tumor; and as to the effect of radiotherapy, only one report (5) could be found. It showed that a multimodality treatment consisting of conservative resection plus iridium-192 implants (40 Gy) plus external radiation therapy ( $30 \mathrm{~Gy}$ ) was very effective in two such cases; one was a case with a tumor in the left calf and popliteal space, and the other was a case with a tumor in the buttock and pelvic region. Information on the effect of radiotherapy is extremely limited, so we can not conclude that EMC is generally a radiosensitive tumor. Accumulation of these cases will be required to draw a conclusion. At any rate, we think it is worthwhile to try radiotherapy for this disease.

The rates of recurrence or metastasis after surgical resection of EMC are reported to be relatively high, and the common metastatic site is shown to be in the lung as observed in this case. However, this case seems very unique, because no reports could be found showing obstruction of the bronchus. We decided to apply the Nd-YAG laser treatment first as an emergency measure, because this patient could not sleep well in the supine position. Treatment with Nd-YAG laser alone was not effective to open the lumen of the right main bronchus, because this tumor was bone-like hard. Thus Nd-YAG laser and biopsy forceps were both required. This may be the first report showing the therapeutic effect of Nd-YAG laser on this type of tumor. Complete control by means of Nd-YAG laser is usually impossible for solid tumors, therefore we applied radiation therapy although we were not sure that it would be effective. The orifice of the right upper lobe had remained closed. Concerning this point, we speculate that this might be due to the adhesion of the orifice. EMC is a slow-growing tumor and has a relatively good prognosis. Indeed, the present patient survived to the age of 81 years old with a 14-year history of this disease. We think that radiation therapy should be tried for the control of its local spread.

\section{References}

1) Stout AP, Verner EW. Chondrosarcoma of the extraskeletal soft tissues. Cancer 6: 581, 1953.

2) Enzinger FM, Shiraki M. Extraskeletal myxoid chondrosarcoma: an analysis of 34 cases. Hum Pathol 3: 421, 1972.

3) Goldenberg RR, Cohen P, SteinlaufP.Chondrosarcoma of the extraskeletal soft tissues: a report of seven cases and review of the literature. J Bone Joint Surg (Am) 49: 1487, 1967.

4) Tsuneyoshi M, Enjoji M, Iwasaki H, Shinohara N. Extraskeletal myxoid chondrosarcoma: a clinicopathologic and electron microscopic study. Acta Pathol Jpn 31: 439, 1981.

5) D'Ambrosio FG, Shiu MH, Brennan MF. Intrapulmonary presentation of extraskeletal myxoid chondrosarcoma of the extremity: report of two cases. Cancer 58: 1144, 1986.

6) Fletcher CD, Powell G, McKee PH. Extraskeletal myxoid chondrosarcoma: a histochemical and immunohistochemical study. Histopathol 10: 489, 1986.

7) Saleh G, Evans HL, Ro JY, Ayala AG. Extraskeletal myxoid chondrosarcoma: a clinicopathologic study of ten patients with long-term followup. Cancer 70: 2827, 1992.

8) Patel SR, Burgess MA, Papadopoulos NE, Linke KA, Benjamin RS. Extraskeletal myxoid chondrosarcoma: long-term experience with chemotherapy. Am J Clin Oncol 18: 161, 1995.

9) Hirabayashi $Y$, Ishida T, Yoshida MA, etal.Translocation (9;22)(q22,q12), a recurrent chromosome abnormality in extraskeletal myxoid chondrosarcoma. Cancer Genet Cytogenet 81: 33, 1995. 